\title{
Image in image Steganography Technique based on Arnold Transform and LSB Algorithms
}

\author{
Sadik Ali Al-Taweel \\ Information Systems Department \\ University science \& Technology \\ Sana'a, Yemen
}

\author{
Mohammed Husain Al-Hada \\ Computer Science Department \\ University science \&Technology \\ Sana'a, Yemen
}

\author{
Ahmed Mahmoud Nasser \\ Computer Science Department \\ University science \& Technology \\ Sana'a, Yemen
}

\begin{abstract}
Computer security, for quite a long time, was almost a limited field of study which was mainly the concern of theoretical computer scientists, electrical engineers, and applied mathematicians. The techniques of concealing data have taken an important role with the rapid growth of intensive transfer of multimedia content and secret communications. There are many techniques used for data hiding and the well-known technique is the Steganography. Steganography is the art of concealing information in ways that prevent detection. For hiding secret information in images, there exists a large variety of Steganography techniques, some are more complex than others and all of them have respective strong and weak points. This paper deals with encrypt and hiding an image in another gray image file using Least Significant Bit (LSB) based Steganography and Arnold's transformation algorithm based Cryptography. This algorithm will be against Noise, Sharpening and Contrast Attacks. Experimental results indicate that the algorithm has good security and imperceptibility in grayscale images.
\end{abstract}

\section{General Terms}

Digital Image Processing, Steganography, Security

\section{Keywords}

Image processing, Steganography, information hiding, Arnold Transform

\section{INTRODUCTION}

Based on [1] it was stated that "Security through obscurity says that if you hide the inner workings of your system you will be secure. This philosophy does not work when it comes to security, and it does not work when it comes to cryptography". Most of the requirements of secret communication, sometimes in combination with other techniques, such as cryptography, and Steganography, complement each other. It is recommended to use these two techniques together to obtain a higher level of security.

Information security is the protection of information and the systems and hardware that use, store, and transmit that information. It can be defined as measures adopted to prevent the unauthorized use or modification of using data or capabilities [2].

Image scrambling refers to some kind of transform, which makes the spatial location of the pixel become chaotic, and lose their original properties and meaning. However, the total number of pixels and histogram has not unchanged so as to achieve the purpose of encryption. In addition, the scrambling must be one kind of reversible transformation; otherwise it will not have any significance in the practical application. If anyone does not know the rules and keys of the transformation, it is impossible to retrieve the original image. During the process of scrambling, the loss of the hidden information is also dispersed into the whole hidden data, where it minimizes the loss of meaningful information so as to reach the purpose of improving robustness. Therefore, image scrambling technology has been widely applied in the image Steganography field [3].

Arnold transform is a type of image scrambling method. The transformation shifts pixel position from $(\mathrm{x}, \mathrm{y})$ to $\left(\mathrm{x}^{\prime}, \mathrm{y}^{\prime}\right)$ without changing its gray value. It is cyclic which means the secret image repeats itself after a certain number of iteration.

\section{RELATED WORK}

M. Mishra, et.al [4] propose a spatial domain LSB substitution method for information embedding and Arnold's transform is applied twice in two different phases to ensure security. The proposed system is checked and validated against a series of standard images and it does not resist noise, as the lower order bit planes are generally affected by noises and compression techniques.

P. Das, et al [5] suggested hiding multiple secret images in a single 24-bit cover image using LSB substitution based image steganography. They have encrypted a secret image before hiding in the cover image using Arnold Transform.

S. Shankar, et al [6] proposed data hiding and image encryption scheme using random diffusion and two dimensional Arnold cat mapping transform. They also placed secret message bits in the least significant bit positions of the cover image. The shared key is used to generate 8 bit random integer stream and also added to the stego image in the random diffusion step.

M. Mahdavi, et.al [7] suggested a new accurate steganalysis method for the LSB replacement Steganography. The proposed method is based on the changes that appear in the histogram of an image after the incorporating of data. Every pair of adjacent bins of a histogram are either inter-related or unrelated depending on whether embedding of a bit of data in the image could affect both bins or not.

Chang, C.C et al [8] proposed an image Steganography technique which offers high embedding capacity and brings less distortion to the stego image. The process of embedding embeds bits of secret bit stream on the stego image pixels. Instead of replacing the LSB of every pixel, this method replaces the pixel intensity with a similar value. The range of modifiable pixel value is higher in edge areas than smooth areas to maintain good perceptual excellence. This method has a shortcoming which is a boundary problem; this means the pixel which is located for embedding will become unused; since it exceeds the maximum intensity level that is greater than 255 (maximum gray scale intensity). 
Wu. H.C et al [9] proposed an improvement in the capacity of the hidden secret data so as to provide an imperceptible Stego-image quality. This method is based on the least significant bit (LSB) replacement and the pixel-value differencing (PVD) method is also suggested in this paper. The limitation of this method is Low-hiding capacity which is attributed to mainly hiding in smooth areas. For example if a pixel value difference is 3 of the corresponding range width is 8 , only 3 bits can be embedded in a pair of pixels.

Y. K. Jain et al [10] proposed an adaptive least significant bit spatial domain embedding method. This method divides the image pixels ranges (0-255) and generates a stego-key. This private stego-key has 5 different gray level ranges of image and each range refers to substitute fixed number of bits to embed in least significant bits of image. The strength of this method is its integrity of secret hidden information in stegoimage and high hidden capacity. This method could be weak for hiding extra bits of signature with a hidden message for its integrity purpose. This study also proposed a method for color image just to modify the blue channel with this scheme for information hiding. The purpose of this method is to achieve high hidden capacity plus security of a hidden message.

According to Yang et al., in [11], an adaptive LSB substitution based data hiding method for image is proposed for achieving a better visual quality of stego-image. The noise sensitive area for embedding is taken care of by this method.. The proposed method differentiates and takes advantage of normal texture and edges area for embedding. Using this method one can analyze the edges, brightness and texture masking of the cover image to calculate the number of k-bit LSB for secret data embedding. The value of $k$ becomes high at non-sensitive image region and over sensitive image area (k) value remains small in order to balance the image overall visual quality. The LSB's $(\mathrm{k})$ for embedding is calculated by the high-order bits of the image. It also uses the pixel adjustment method for better stego-image visual quality through LSB substitution method. The overall result reveals a good high hidden capacity, but dataset for experimental results are limited. There is no single image which has many edges with noise region like 'Baboon.tif'.

C.-H. Yang et al [12] proposed that a Pixel Value Difference (PVD) and simple least significant bits scheme are used to achieve adaptive least significant bits data embedding. In PVD, the size of the hidden data bits can be estimated by the difference between the two consecutive pixels in cover image using simple relationship between two pixels. This method generally provides a good imperceptibility by calculating the difference of two consecutive pixels which determine the depth of the embedded bits. The proposed method hides large and adaptive k-LSB substitution at edge area of image and PVD for smooth region of image. In this way, therefore, the technique provides both larger capacity and high visual quality according to experimental results. However, the algorithm proposed by them seems complex because of adaptive (k) generation for substitution of LSB

K.-H. Jung et al [13] proposed a method of Multi-Pixel Differencing (MPD) which uses more than two pixels to estimate smoothness of each pixel for data embedding and it computes the sum of difference value of four pixels block. For a small difference value, it uses the LSB, whereas for a high difference value it uses the MPD method for data embedding. In this method the experimental dataset is too limited.
In [14] the authors suggested another pixel value differencing method, which used three pixels for data embedding near the target pixel. Also, it uses the simple k-bit LSB method for secret data embedding where the number of k-bit is estimated by near three pixels with a high difference value. To keep better visual quality and high capacity, it simply uses the optimal pixel adjustment method on target pixels. In this method, the histogram of stego-image and cover-image are almost the same, but the dataset for experiments is too small

W. J. Chen et al [15] introduced a high capacity of hidden data utilizing the LSB and hybrid edge detection scheme. For edge calculation, two types of canny and fuzzy edges detection method were applied and simple LSB substitution was used to embed the hidden data. This scheme is successful to embed data with a higher peak signal to noise ratio (PSNR) with normal LSB based embedding. This method is tested on limited images dataset.

Based on LSB substitution and selection of random pixel of required image area, Madhu et al., in [16] proposed an image steganography method. The purpose of this method is to improve the security where password is added by LSB of pixels. It also generates the random numbers and selects the region of interest where a secret message has to be hidden. The limitation of this method is that it does not consider any type of perceptual transparency.

\section{PROPOSED ALGORITHM}

Based on [17] the methodology of proposed method is given as follows:

\subsection{Arnold Transform and LSB Algorithms}

Before embedding, the secret message is implemented for block transformation using the Arnold image transformation. The Arnold image transformation is defined as the point (x,y) in the unit square transforms into the other point $\left(\mathrm{x}^{\prime}, \mathrm{y}^{\prime}\right)$ [18]:

$\left[\begin{array}{l}\dot{x} \\ y\end{array}\right]=\left[\begin{array}{ll}1 & 1 \\ 1 & 2\end{array}\right]\left[\begin{array}{l}x \\ y\end{array}\right](\bmod N)$

Where, $\left(x^{\prime}, y^{\prime}\right)\{0,1,2,3 \ldots N-1\}$ are pixel coordinates of the secret image, $(\mathrm{x}, \mathrm{y})$ is the transformed position of $\left(\mathrm{x}^{\prime}, \mathrm{y}^{\prime}\right)$ and $\mathrm{N}$ is the order number of image matrix. Suppose the secret image has iterated for $\mathrm{K}$ iterations then the "chaotic" secret image will be obtained; so K can be saved as a key1. Figure1 shows an example of Arnold transform with four iterations.
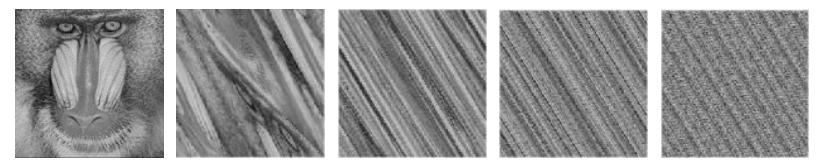

Fig 1: Example of Arnold Transform. The first image is the secret image, which has been encrypted with four iterations separately as shown.

After the encryption step the secret will hide in cover image by using Least Significant Bit (LSB) Replacement. This is the simplest method of the steganography methods which are based on the use of LSB, and therefore the most vulnerable. The embedding process consists of the sequential replacement of each Least Significant Bit (LSB-1-2) of the image pixel for the bit-stream of secret image by bit-or function. The extracting process also consists of sequential extracting for bit-stream of secret image by concatenation method. For its simplicity, this method can hide a great volume of information. Figure 2 shows the diagram of the proposed 
method and the following algorithm steps illustrate how the proposed method works.

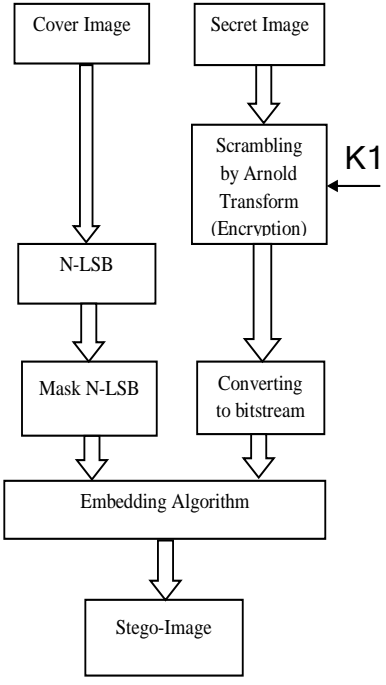

A

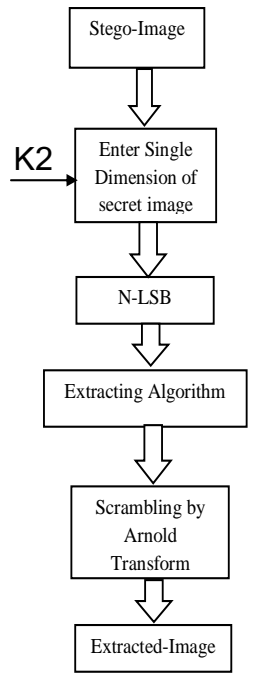

B
Fig 2: Block Diagram of Proposed Method: (A) Embedding Algorithm, (B) Extracting Algorithm.

In this figure, there are two keys $\mathrm{K} 1$ is a key of Arnold transform algorithm which will be the number of iterations. Moreover, K2 is a key of proposed LSB algorithm, when you want to extract the secret image you must enter a single dimension of the secret image. By this number of dimension you can extract the right secret image. So, it will be another key and saved as K2.

\subsection{Encryption and Embedding Algorithm}

1. Read Cover image and Secret image.

2. Enter the number of iterations for encrypt the secret image (K1).

3. Apply Image scrambling Algorithm using Arnold Transform Method.

4. Enter the $\mathrm{N}$ bit plane that will be hide in.

5. Mask N-LSB of image pixel in cover.

6. Convert Secret image to bit stream.

7. If size of bits of secret image is bigger than the total size of the bit space, insert-able show error message and read another secret image; otherwise continue.

8. Hide first $\mathrm{N}$ bit of bit stream in Masked Last $\mathrm{N}$ of covered image.

9. Repeat step 6 until all the bits of bit stream are embedded.

10. Then create stego image.

\subsection{Decryption and Extracting Algorithm}

1. Read Stego image.

2. Enter the single dimension of the secret image (K2).

3. Enter the N-LSB which is hidden in

4. Find the Length of embedded bitstream.

5. Create a new bit stream.

6. Convert Stego image from decimal to binary.

7. Concatenate the new bit stream and Last $\mathrm{N}$ bit of binary Stego image.

8. Convert the new bitstream to array.

9. Then retrieve the scrambled image.

10. Enter the number of iterations for decrypt the secret image (K1).

11. Apply Image scrambling Algorithm using Arnold Transform Method.
12. Finally, recover the secret image.

\section{RESULT AND DISCUSSION}

In Steganography, the technique Peak Signal-to-Noise Ratio (PSNR), Normalized Correlation (NC) is a standard measure used in order to test the quality of the stego images. The PSNR is used to evaluate the imperceptibility of the Stegoimage, the maximum value is (100) and the minimum value is (0), whenever the bigger, the better. It can be found in equation (2). MSE is the Mean Square Error. For imperceptible hiding, the stego-image should appear as similar as the cover-image, whenever was the youngest, the better. It can be found in equation (3). In this section, some experiments are carried out to demonstrate the efficiency of the proposed method without and with attack. Before the embedding process, the secret image was firstly encrypted using Arnold transform algorithm. Three of 8-bit grayscale images of size $512 * 512$ used as cover and shown in Figure 3 .

$$
\begin{gathered}
M S E=\frac{1}{M x N} \sum_{i=0}^{M} \sum_{j=0}^{N}\left(I_{B}(i, j)-I_{H}(i, j)\right)^{2} \\
P S N R=10 x \log _{10}\left(\frac{255^{2}}{M S E}\right)(d b)
\end{gathered}
$$

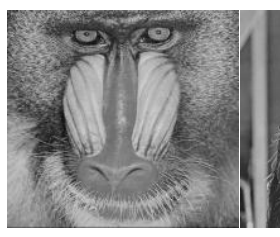

(A)

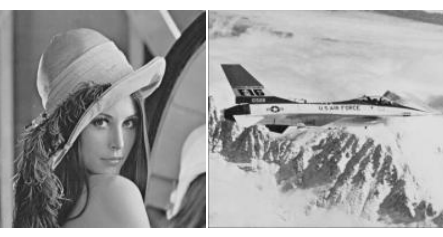

(B)
(C)
Fig 3: Cover Images: (A) Baboon (B) Lena (C) Airplane

\subsection{Results without Attack}

In this section, the proposed method has been tested in three experiments without attack by taking three standard $512 * 512$ gray scale images (Baboon, Lena and Airplane) as a cover and three secret images with different lengths. Figure 4 used "Baboon.bmp" as a Cover image and "Flower.bmp" as Secret image with size (128*128). And Figure 5 used "Lena.bmp" as Cover and "Baboon.bmp" as Secret image with size (192*192). Also Figure 6 used "Airplane.bmp" as a cover image and "Lena.bmp" as a Secret image with size (256*256).

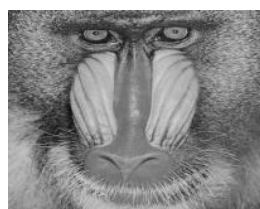

(A)

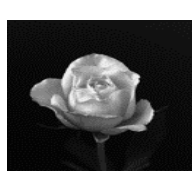

(B)

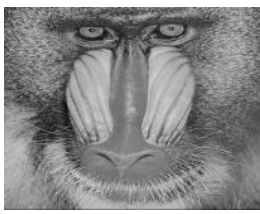

(C)
Fig 4: First Embedding Experiment: (A) Cover image, (B) Secret Image, (C) Stego-Image

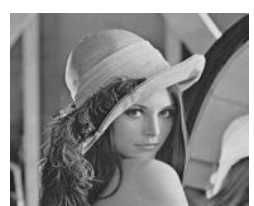

(A)

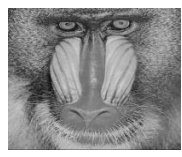

(B)

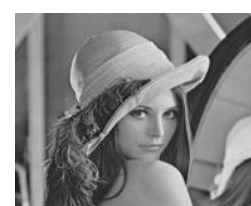

(C)
Fig 5: Second Embedding Experiment: (A) Cover image, (B) Secret Image, (C) Stego-Image. 


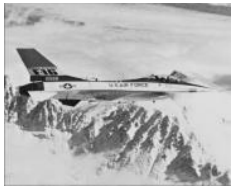

(A)

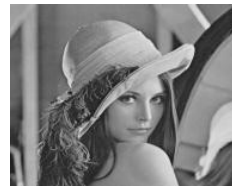

(B)

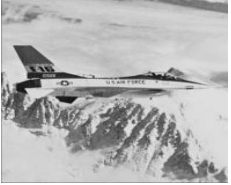

(C)
Fig 6: Third Embedding Experiment: (A) Cover image, (B) Secret Image, (C) Stego-Image.

\subsubsection{PSNR}

The results that are obtained from these experiments are recorded and compared with another method in [19]. It can be summarized in the following table:

Table 1. Comparative Performance of MSE, PSNR without Attack

\begin{tabular}{|l|l|l|l|}
\hline Methods & $\begin{array}{l}\text { Cover Image } \\
(\mathbf{5 1 2} * \mathbf{5 1 2})\end{array}$ & $\begin{array}{l}\text { Number of } \\
\text { Hidden Bits }\end{array}$ & PSNR \\
\hline \multirow{3}{*}{ LSB SM } & Lena & 164538 & 38.56 \\
\cline { 2 - 4 } & Baboon & 298413 & 48.18 \\
\hline \multirow{3}{*}{$\begin{array}{l}\text { Proposed } \\
\text { Method }\end{array}$} & Lena & 294912 & 43.60 \\
\cline { 2 - 4 } & Baboon & 131072 & 51.13 \\
\cline { 2 - 4 } & Airplane & 524288 & 44.29 \\
\hline
\end{tabular}

As shown in the table the proposed method has been compared with another method, which is labeled (LSB SM) with different capacity for the embedding. The proposed Method has three experiments, while the other method has two experiments. Here PSNR is used for indicate the preference. The following histogram will show the different rate of the two methods.

\subsubsection{The Simulation Results of Performance} Measurement

The measurement of the quality for a stego image, the Peak Signal to Noise Ratio (PSNR) was used in the performance measurement of the proposed method. The proposed method was compared with the existing methods and the quality of the stego image.

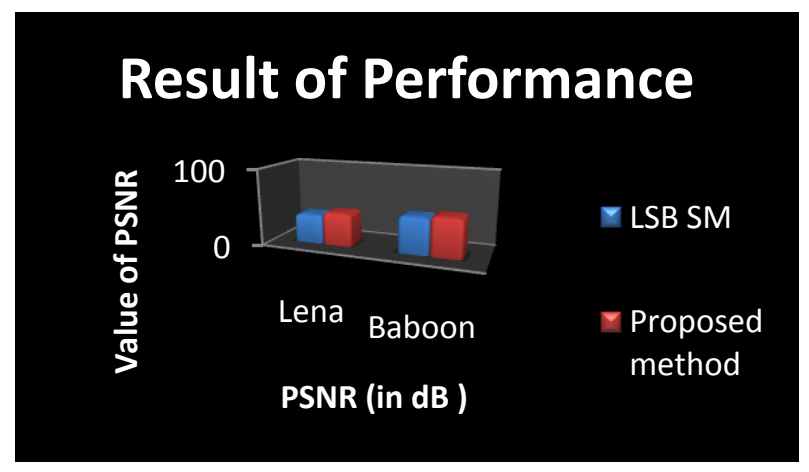

Fig 7: Comparative of PSNR

\subsubsection{NC}

The results of NC obtained from secret images are shown in Figures 8, 9 and 10 below and recorded in the next table:

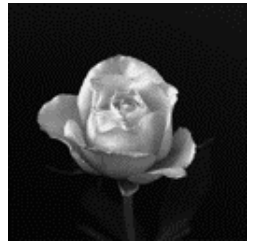

( A)

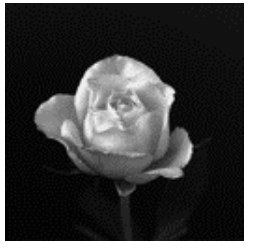

(B)
Fig 8: First NC without Attack: (A) Secret Image $(128 * 128)$, (B) Extracted Image $(128 * 128)$.

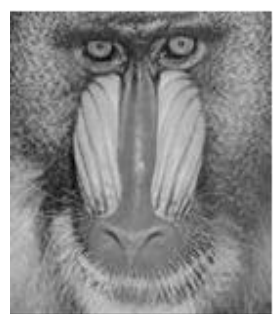

(A)

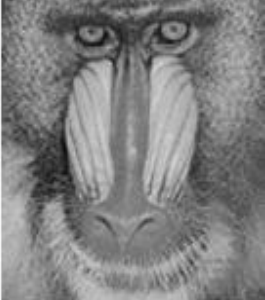

(B)
Figure 9: Second NC without Attack: (A) Secret Image (192*192), (B) Extracted Image (192*192).

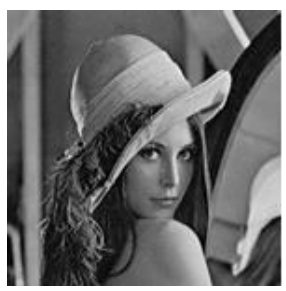

(A)

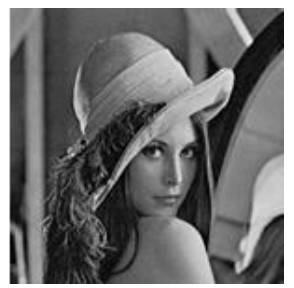

(B)
Fig 10: Third NC without Attack: (A) Secret Image (256*256), (B) Extracted Image (256*256).

Table 2. Result of NC without Attack

\begin{tabular}{|l|l|l|}
\hline Secret Image & Extracted Image & NC \\
\hline Flower $(128 * 128)$ & Flower $(128 * 128)$ & 1 \\
\hline Baboon $(192 * 192)$ & Baboon $(192 * 192)$ & 1 \\
\hline Lena $(256 * 256)$ & Lena $(256 * 256)$ & 1 \\
\hline
\end{tabular}

The table shows the results that are similar to $\mathrm{NC}$ values which are obtained from the different experiments of coverimages in Figures 8,9 and 10, which are graphed in the following histogram.

\subsubsection{Histogram of $\mathrm{NC}$}

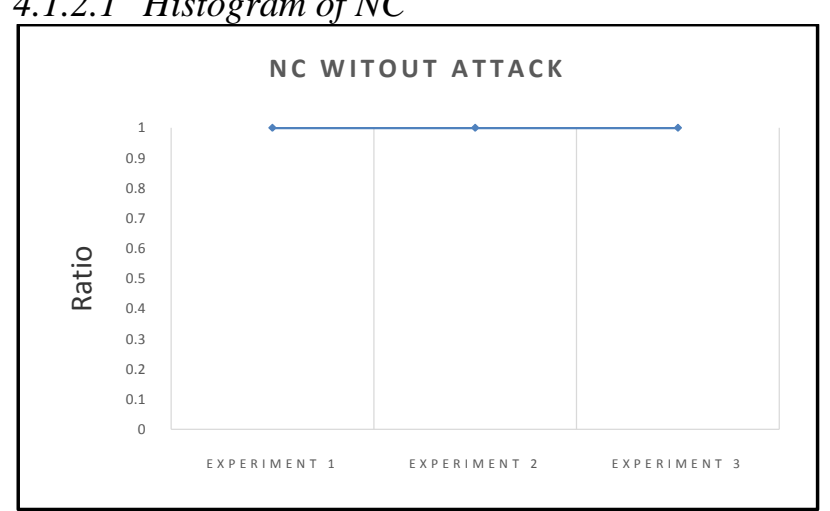

Fig 11: Histogram of NC without Attack. 


\subsection{Results with Attack}

In this section, the proposed method was tested in three experiments by using three types of attacks with different values. Three gray scale 512*512 images (Baboon, Lena and Airplane) were used as a cover and two gray scale images (Flower and Baboon) were used as secret images with different length for each.

\subsubsection{Noise Attack}

This section will test the proposed method using noise attack with three values. Figure 12 shows ("Baboon.bmp", "Lena.bmp" and "Airplane.bmp") images before/after attack. Baboon was tested with value (0.03), Lena was tested with value (0.04) and Airplane was tested with value (0.05).

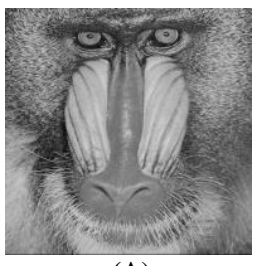

(A)

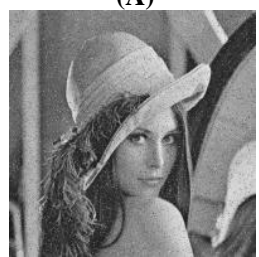

(D)

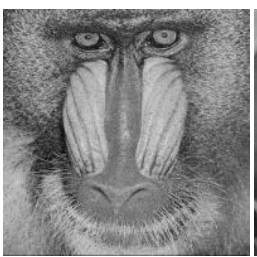

(B)

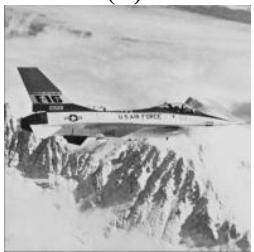

(E)

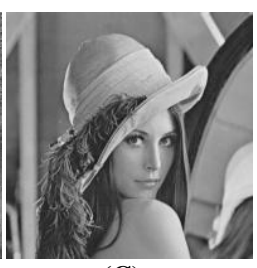

(C)

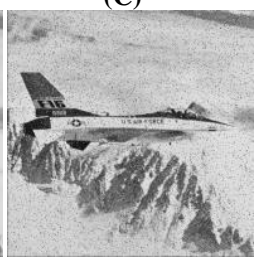

(F)
Figure 12. Noise Attack: (A) Baboon before noise attack, (B) Baboon after noise attack, (C) Lena before noise attack, (D) Lena after noise attack, (E) Airplane before noise attack, (F) Airplane after noise attack.

\subsubsection{Sharpening Attack}

This section will test the proposed method using sharpening attack with three values. Figure 13 shows ("Baboon.bmp", "Lena.bmp" and "Airplane.bmp") images before and after attack. Baboon tested with value (0.00001), Lena tested with value (0.00002) and Airplane tested with value (0.00003).

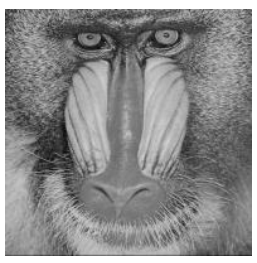

(A)

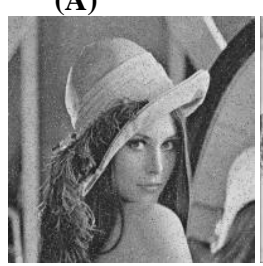

(D)

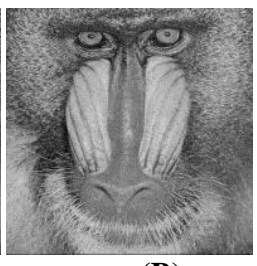

(B)

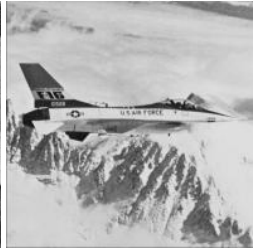

(E)

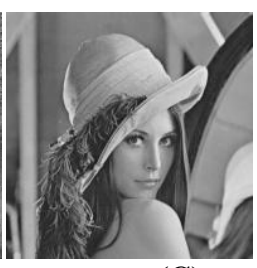

(C)
Figure 13.:Sharpening Attack: (A) Baboon before sharpening attack, (B) Baboon after sharpening attack,

(C) Lena before sharpening attack, (D) Lena after sharpening attack, (E) Airplane before sharpening attack, (F) Airplane after sharpening attack.

\subsubsection{Contrast Attack}

This section will test the proposed method using sharpening attack with three values. Figure 14 Shows ("Baboon.bmp" "Lena.bmp" and "Airplane.bmp") images before/after attack.

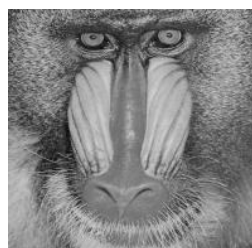

(A)

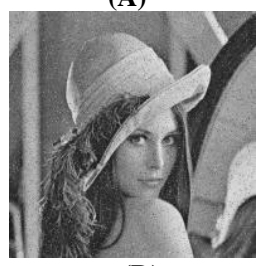

(D)

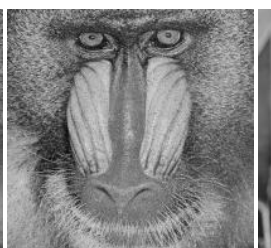

(B)

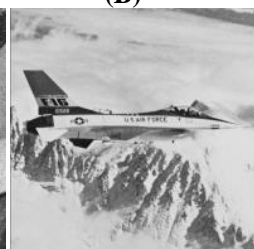

(E)

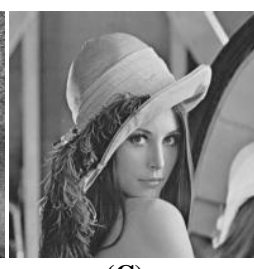

(C)

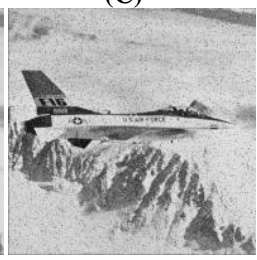

(F)
Figure 14: Contrast Attack: (A) Baboon before Contrast attack, (B) Baboon after Contrast attack,

(C) Lena before Contrast attack, (D) Lena after Contrast attack, (E) Airplane before Contrast attack,

(F) Airplane after Contrast attack.

\subsubsection{MSE-PSNR with Attack}

Tables 3,4 and 5. Figures 15, 16 and 17 show the results that similar PSNR values which obtained from the different experiments with three types of attacks in different cover and secret images.

Table 3. Results of MSE and PSNR with Noise attack.

\begin{tabular}{|l|l|l|l|l|}
\hline $\begin{array}{l}\text { Cover } \\
\text { Image }\end{array}$ & $\begin{array}{l}\text { Ratio of } \\
\text { Attack }\end{array}$ & $\begin{array}{l}\text { Size of } \\
\text { Capacity }\end{array}$ & MSE & PSNR \\
\hline Baboon & 0.03 & 32768 & 533.602 & 20.858 \\
\hline Lena & 0.04 & 131072 & 746.753 & 19.399 \\
\hline Airplane & 0.05 & 294912 & 1084.763 & 17.777 \\
\hline
\end{tabular}

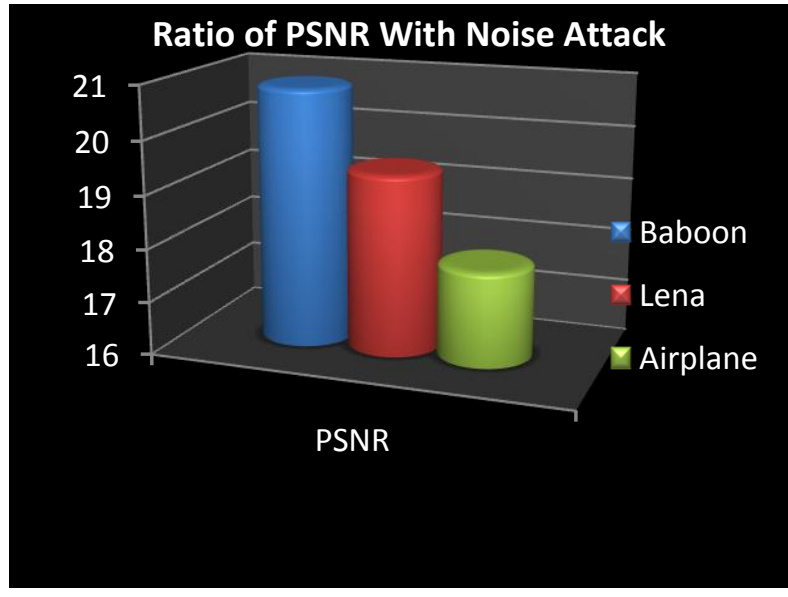

Figure 15: PSNR with Noise Attack 
Table 4. Results of MSE and PSNR with Sharpening attack.

\begin{tabular}{|l|l|l|l|l|}
\hline over Image & $\begin{array}{l}\text { Ratio } \\
\text { of } \\
\text { Attack }\end{array}$ & $\begin{array}{l}\text { Size of } \\
\text { Capacity }\end{array}$ & MSE & PSNR \\
\hline Baboon & 0.00001 & 32768 & 533.602 & 20.858 \\
\hline Lena & 0.00002 & 131072 & 746.753 & 19.399 \\
\hline Airplane & 0.00003 & 294912 & 1084.763 & 17.777 \\
\hline
\end{tabular}

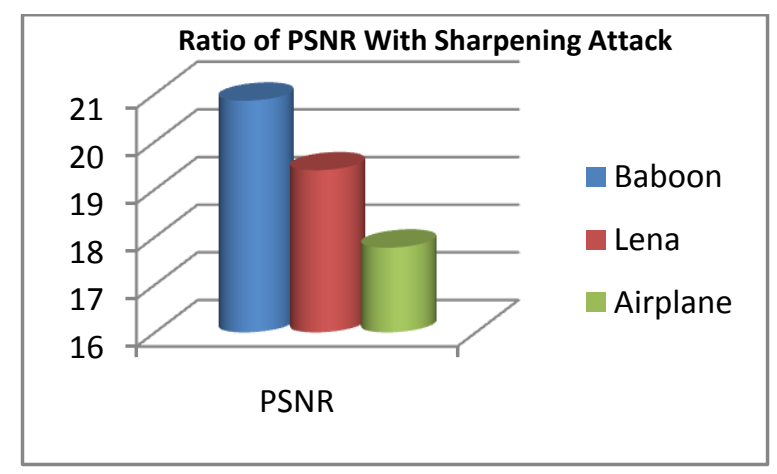

Figure 16: PSNR with Sharpening Attack

Table5. Results of MSE and PSNR with Contrast attack.

\begin{tabular}{|l|l|l|l|l|}
\hline $\begin{array}{l}\text { Cover } \\
\text { Image }\end{array}$ & $\begin{array}{l}\text { Ratio of } \\
\text { Attack }\end{array}$ & $\begin{array}{l}\text { Size of } \\
\text { Capacity }\end{array}$ & MSE & PSNR \\
\hline Baboon & 0 & 32768 & 1121.787 & 17.631 \\
\hline Lena & 0 & 131072 & 800.249 & 19.098 \\
\hline Airplane & 0 & 294912 & 4383.738 & 11.712 \\
\hline
\end{tabular}

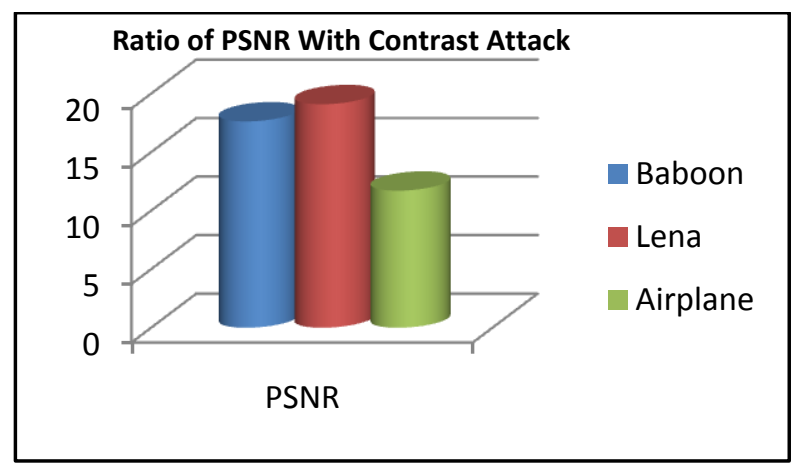

Figure 17: PSNR with Contrast Attack

From the above Tables and figures the results show that similar PSNR values were obtained from the different experiments with three types of attack in different cover and secret images. In the following the histogram of the obtained values is given.

\subsubsection{NC}

Here, NC has been computed by using three types of attacks (Noise attack, Sharpening Attack and Contrast Attack). Two gray scale images (Flower and Baboon) were used as secret images. The first image (Flower) with size $64 * 64$, second (Baboon) with size $128 * 128$ and the third image (Baboon) with size $192 * 192$. The results of NC that were obtained from secret images are shown in Figures $(18,19$, and 20) below and recorded in the next Table:

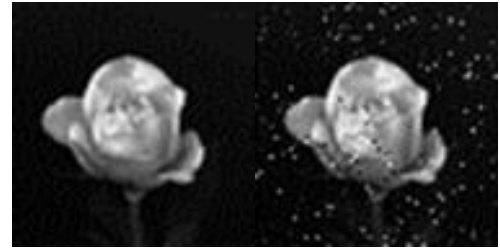

(A)

(B)

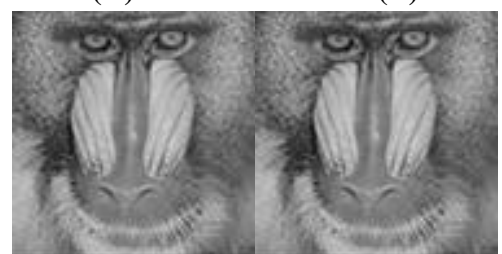

(C)

(D)

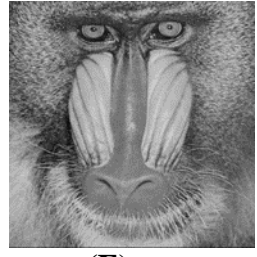

(E)

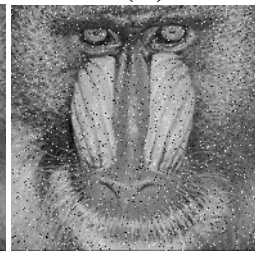

(F)
Figure 18: Experiments (NC) Noise Attack.

(A) Flower before Noise attack, (B) Flower after Noise attack, (C) Baboon size 128*128 before Noise attack, (D) Baboon size $128 * 128$ after Noise attack, (E) Baboon size $192 * 192$ before Noise attack, (F) Baboon size 192*192 after Noise attack.

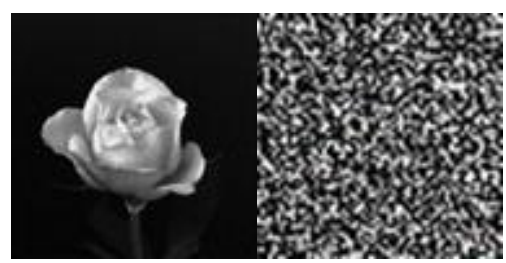

(A) (B)

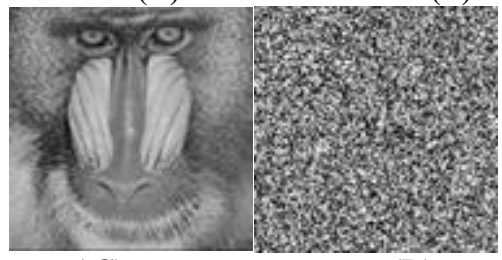

( C) (D)

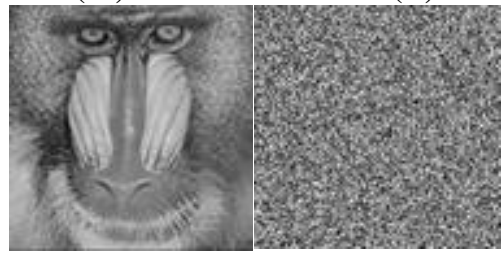

(E)

(F)

Figure 19: Experiments (NC) Sharpening Attack.

(A) Flower before Sharpening attack, (B) Flower after Sharpening attack, (C) Baboon size $128 * 128$ before Sharpening attack, (D) Baboon size $128 * 128$ after Noise Sharpening attack, (E) Baboon size 192*192before Sharpening attack, (F) Baboon size 192*192 after Sharpening attack. 


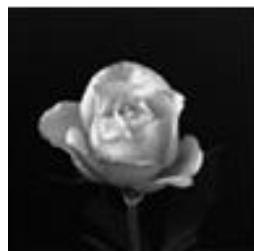

(A)

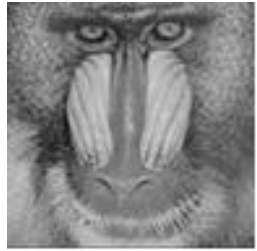

(C)

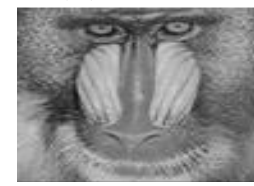

(E)

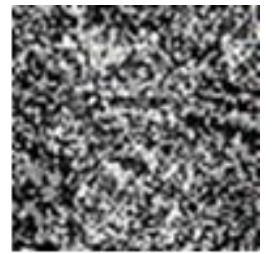

(B)

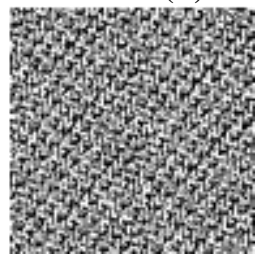

(D)

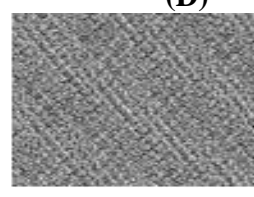

(F)
Figure 20: Experiments (NC) Contrast Attack.

A) Flower before Contrast attack, (B) Flower after Contrast attack, (C) Baboon size $128 * 128$ before Contrast attack, (D) Baboon size $128 * 128$ after Contrast Sharpening attack, (E) Baboon size 192*192before Contrast attack, (F) Baboon size 192*192 after Contrast attack.

All experiments of $\mathrm{NC}$ with attack have been obtained with different values for each attack. The following Table and Figure show the type of attacks with three ratios for each attack and (secret \& extracted) images size.

Table 6.Result of NC with Attacks

\begin{tabular}{|c|c|c|c|c|}
\hline Attacks & $\begin{array}{l}\text { Ratio of } \\
\text { Attack }\end{array}$ & $\begin{array}{l}\text { Secret } \\
\text { Image }\end{array}$ & $\begin{array}{l}\text { Extracted } \\
\text { Image }\end{array}$ & $\mathrm{NC}$ \\
\hline \multirow{3}{*}{ Noise } & 0.03 & $\begin{array}{l}\text { Flower } \\
(64 * 64)\end{array}$ & $\begin{array}{l}\text { Flower } \\
(64 * 64)\end{array}$ & 0.970 \\
\hline & 0.04 & $\begin{array}{l}\text { Baboon } \\
(128 * 128)\end{array}$ & $\begin{array}{l}\text { Baboon } \\
(128 * 128)\end{array}$ & 0.988 \\
\hline & 0.05 & $\begin{array}{l}\text { Baboon } \\
(192 * 192)\end{array}$ & $\begin{array}{l}\text { Baboon } \\
(192 * 192)\end{array}$ & 0.983 \\
\hline \multirow{3}{*}{ Sharpening } & 0.00001 & $\begin{array}{l}\text { Flower } \\
(64 * 64)\end{array}$ & $\begin{array}{l}\text { Flower } \\
(64 * 64)\end{array}$ & 0.490 \\
\hline & 0.00002 & $\begin{array}{l}\text { Baboon } \\
(128 * 128)\end{array}$ & $\begin{array}{l}\text { Baboon } \\
(128 * 128)\end{array}$ & 0.833 \\
\hline & 0.00003 & $\begin{array}{l}\text { Baboon } \\
(192 * 192)\end{array}$ & $\begin{array}{l}\text { Baboon } \\
(192 * 192)\end{array}$ & 0.831 \\
\hline \multirow{3}{*}{ Contrast } & & $\begin{array}{l}\text { Flower } \\
(64 * 64)\end{array}$ & $\begin{array}{l}\text { Flower } \\
(64 * 64)\end{array}$ & 0.445 \\
\hline & & $\begin{array}{l}\text { Baboon } \\
(128 * 128)\end{array}$ & $\begin{array}{l}\text { Baboon } \\
(128 * 128)\end{array}$ & 0.731 \\
\hline & & $\begin{array}{l}\text { Baboon } \\
(192 * 192)\end{array}$ & $\begin{array}{l}\text { Baboon } \\
(192 * 192)\end{array}$ & 0.836 \\
\hline
\end{tabular}

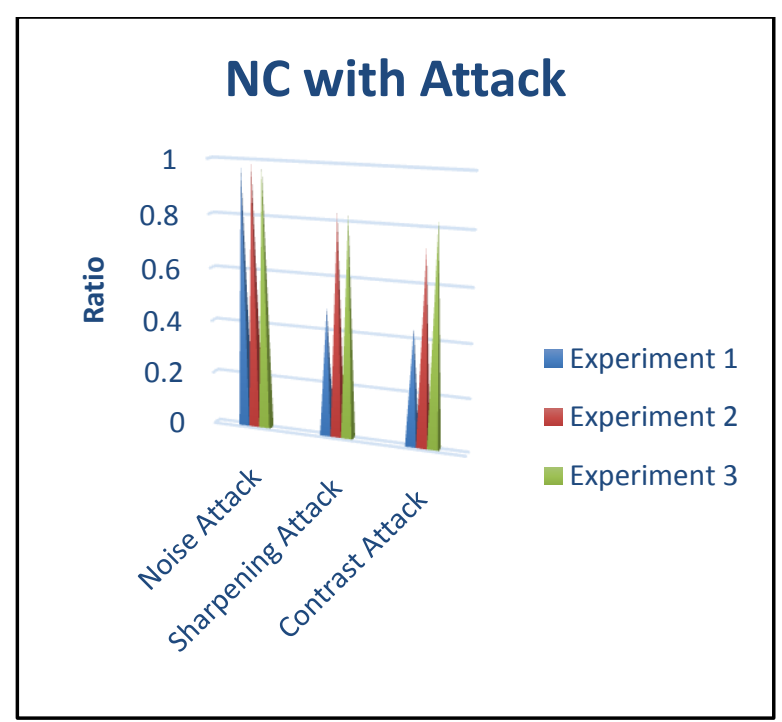

Figure 21: Histogram of NC with Attacks.

\section{CONCLUSION}

The proposed method described in this paper helps to successfully hide the secret image into the cover image, with minimum distortion made to the cover image. First, the secret image was scrambled using Arnold algorithm and embedded to the cover image by using LSB algorithm. This method is essential for the construction of accurate targeted and blind Steganalysis methods for BMP images. By using Arnold transform, the proposed method will be more secure. The main features of the proposed method are imperceptibility and security. One limitation of the proposed method is being slow in extracting algorithm when using a large size image higher than 160*160. Also, it cannot use images which have different dimensions; it can only use a square array of image which has the same dimensions.

\section{REFERENCES}

[1] E. Cole. 2003. Hiding in Plain Sight: Steganography and the Art of Covert Communication. Indiana, John Wiley \& Sons Inc.

[2] M.E. Whitman \& H.J. Mattord. 2007. Principles of information security. Thomson course technology.

[3] Yinglan Fang, Lin Tian, "An Improved Blind Watermarking Algorithm for Image Based on DWT Domain", Journal of Theoretical and Applied Information Technology, 15th November 2012. Vol. 45 No.1.

[4] M. Mishra, A. Ranjan, s. Kumar, " High Security Image Steganography with Modified Arnold's Cat Map", International Journal of Computer Applications (0975 8887) Volume 37- No.9, January 2012

[5] P.Das, S.C.Kushwaha, M. Chakraborty. 2015. Multiple Embedding Secret Key Image Steganography Using Lsb Substitution And Arnold Transform. IEEE Sponsored 2nd International Conference On Electronics And Communication System (ICECS 2015)

[6] S. Shankar, A. Rengarajan, "Data Hiding In Encrypted Images Using Arnold Transform ", ICTACT Journal On Image And Video Processing, August 2016, Volume: 07, ISSUE: 01 
[7] M. Mahdavi, Sh. Samavi, N. Zaker and M. ModarresHashemi, "Steganalysis Method for LSB Replacement Based on Local Gradient of Image Histogram", Iranian Journal of Electrical \& Electronic Engineering, Vol. 4, No. 3, July 2008.

[8] C.C. Chang, H.W. Tseng. A Steganographic method for digital images using side match. Pattern Recognition Letters 25, 1431-1437, 2004.

[9] H.C. Wu, N. I. Wu, C.S. Tsai, M.S. Hwang. 2004. An Image Steganographic Scheme Based on Pixel-Value Differencing and LSB Replacement Methods. IEEE Proceedings of visual image signal Process, November 7, 2004.

[10] Y. K. Jain and R. R. Ahirwal, "A Novel Image Steganography Method With Adaptive Number of Least Significant Bits Modification Based on Private StegoKeys" International Journal of Computer Science and Security (IJCSS), vol. 4, 2010 March 1.

[11] H. Yang, X. Sun and G. Sun, "A High-Capacity Image Data Hiding Scheme Using Adaptive LSB Substitution", Journal: Radioengineering, vol. 18, no. 4, 2009, pp. 509516

[12] C.H. Yang, C.Y. Weng, S.J. Wang and H.M. Sun, "Adaptive Data Hiding in Edge Areas of Images with Spatial LSB Domain Systems", IEEE Transactions on Information Forensics and Security, vol. 3, no. ( 2008, September 3, pp. 488-497.

[13] K.H. Jung, K. J. Ha and K.Y. Yoo. 2008. Image data hiding method based on multi-pixel differencing and
LSB substitution methods. Proc. 2008. International Conference on Convergence and Hybrid Information Technology (ICHIT '08), Daejeon (Korea), (2008) August 28-30, pp. 355-358.

[14] H. Zhang, G. Geng and C. Xiong. 2009. Image Steganography Using Pixel-Value Differencing. Electronic Commerce and Security, ISECS '09. Second International Symposium on 2009, May.

[15] W. J. Chen, C. C. Chang and T. H. N. Le, "High Payload Steganography Mechanism Using Hybrid Edge Detector" , Expert Systems with Applications (ESWA 2010), vol. 37, pp. 3292-3301, 2010, April.

[16] V. Madhu Viswanatham and J. Manikonda, "A Novel Technique for Embedding Data in Spatial Domain", International Journal on Computer Science and Engineering, IJCSE, vol. 2, 2010.

[17] Sadik. A. Al-Taweel, M. A-Hadam, A.M.Naser, M.AlThamary.2015. Hide Image in Image Based on LSB Replacement and Arnold Transform. The 7th International Conference on Information Technology (ICIT).

[18] John Miano. Compressed image file formats: JPEG, PNG, GIF, XBM, BMP. First printing, July (1999), Copyright $($ ) 1999 by the ACM Press.

[19] Chang, C.C., Tseng, H.W.: "A Steganographic method for digital images using side match", Pattern Recognition Letters 25, 1431-1437 (2004). 